\title{
Droplet dynamics in internally mixed twin-fluid spray
}

\author{
J. Jedelsky \& M. Jicha \\ Energy Institute, Faculty of Mechanical Engineering, \\ Brno University of Technology, Czech Republic
}

\begin{abstract}
Effervescent atomizers are based on the mixing of gas with liquid prior to discharge. We describe the discharge of a two-phase mixture and movement of droplets in a gas jet using simple theoretical models, following with elucidation of droplets dynamics using experimental data for an effervescent spray. Discharge of the liquid-gas mixture from the nozzle is solved using a combination of two discharge models. Depending on operation conditions, 59$64 \%$ of the total discharged mass corresponds to the Separated Flow Model and the rest to the Homogeneous Flow Model. Discharge velocity of the liquid is 12 $27 \%$ of the gas exit velocity. The liquid-gas velocity ratio is negatively correlated with gas-to-liquid mass ratio (GLR) and positively correlated with inlet pressure. Radial profiles of axial droplet velocity, as measured using Phase Doppler anemometry, are axisymmetric bell-shaped with a maximum in the centreline analogous to the profile defined for a simple gas jet which, however, is more flat near the centreline and declines much faster for higher radial positions. Mean velocity in individual spray positions varies with particle size within a range of several $\mathrm{m} / \mathrm{s}$ typically. This variation is closely related to particle Stokes number, Stk. Variation of mean velocity with operation pressure and GLR can be explained with discharge conditions; higher pressures and GLRs lead to higher discharge velocities that are reflected in the spray downstream. Stokes numbers are generally $<<1$ for particle sizes $D_{d}$ up to $10 \mu \mathrm{m}$ so these particles smoothly follow the gas flow. Stk for sizes $10 \mu \mathrm{m}<D_{d}<50 \mu \mathrm{m}$ depends on flow regime and position in the spray and can be found typically within $0.1<$ Stk $<10$. Particles with $D_{d} \geq 100 \mu \mathrm{m}$ have usually Stk $>10$ and very weakly interact with the gas.

Keywords: droplet dynamics, internal mixing, twin-fluid spray, effervescent atomization, Stokes number, velocity field, dispersed two-phase flow.
\end{abstract}




\section{Introduction}

Effervescent atomizers belong to a group of twin-fluid atomizers with internal mixing. Their origin dates back to the early nineties and they have already been used in a number of practical applications, such as combustion of heavy fuels, in gas turbine engines, internal combustion engines, in spray drying processes, spray coating, fire fighting devices, in process industries and others [1].

These applications require the liquid to be delivered as a spray of droplets with well specified size and amount. However velocity of the droplets is usually important as well; in the cases when drop-wall interaction appears or should be avoided and also in reacting sprays (where droplet velocity significantly affects mixing with gas and the flame zone) and in other usages where droplet momentum plays a role.

The process of the spray generation in effervescent atomization consists of several consequent actions: (1) the internal mixing of atomizing gas with atomized liquid, (2) discharge of two-phase gas-liquid mixture, (3) primary breakup of the liquid structures and (4) secondary breakup of formed droplets [2]. Input pressure energy of both the fluids, mainly contained in the energy of the pressurized gas, is transformed into their motion, expansion work, interaction with surrounding air and into increased surface energy of the droplets [3]. All these steps are closely related to the gas-liquid interaction and governed by the expansion of the compressed gas. Break-up mechanism and formation of the spray of internally-mixed atomizers have been intensively studied in past two decades [1, 4-8] however more detailed insight into these processes and into interaction between gas and liquid is important for continuing improvement of the spray characteristics of effervescent atomizers.

We address the issue analysing the gas-liquid interaction in a spray generated by a single-hole effervescent atomizer. Dynamics of droplets is described by means of simple theoretical models and compared with experimental data acquired using Phase Doppler anemometry (PDA).

\section{Methods}

Results, given in the paper, are based on theoretical predictions and also acquired by a measurement of a simple effervescent atomizer by means of PDA. The atomizer experiments were performed on a cold test bench. The atomizer E34 in the "outside-in" gas injection configuration is used in our study. It continuously sprays light heating oil (LHO) with air as an atomizing medium. The atomizer uses a discharge orifice with $2.5 \mathrm{~mm}$ in diameter and mixing chamber $14 \mathrm{~mm}$ in diameter. The air is injected through a set of 24 holes $1 \mathrm{~mm}$ in diameter. For more data about the atomizer, test bench and measurement technique see [9]. Spray characteristics were measured at atomizing pressures, $p$, of $0.1,0.3$ and $0.5 \mathrm{MPa}$ and GLRs of 2,5 and $10 \%$.

Operation of effervescent atomizer is depicted in fig. 1 left. The gas penetrates with low velocity into a liquid flowing through central section of the atomizer. Gas-liquid two-phase flow is created upstream of the atomizer 
discharge orifice. The liquid, reaching the discharge orifice, is transformed by the gas volumes into thin film and ligaments. Gas volumes emerging from the nozzle at significant pressure drop (typically hundreds of $\mathrm{kPa}$ ), expand rapidly and disintegrate the liquid into smaller volumes and finally into droplets. Interaction between the two fluids depends significantly on relative amount of gas and liquid in the mixture (gas to liquid mass ratio, abbrev. GLR).

Typical spray structure is shown in fig. 1 right, where continuous liquid structures emerging the exit orifice change into liquid ligaments, stretch and finally break up due to shear forces. This process leads to a wide and spatially variable size distribution. The droplet size spectrum spans from micron sized droplets to more than one hundred micron drops. Discharged mixture is heterogeneous with significant differences between the velocity of the gas and the liquid phase.
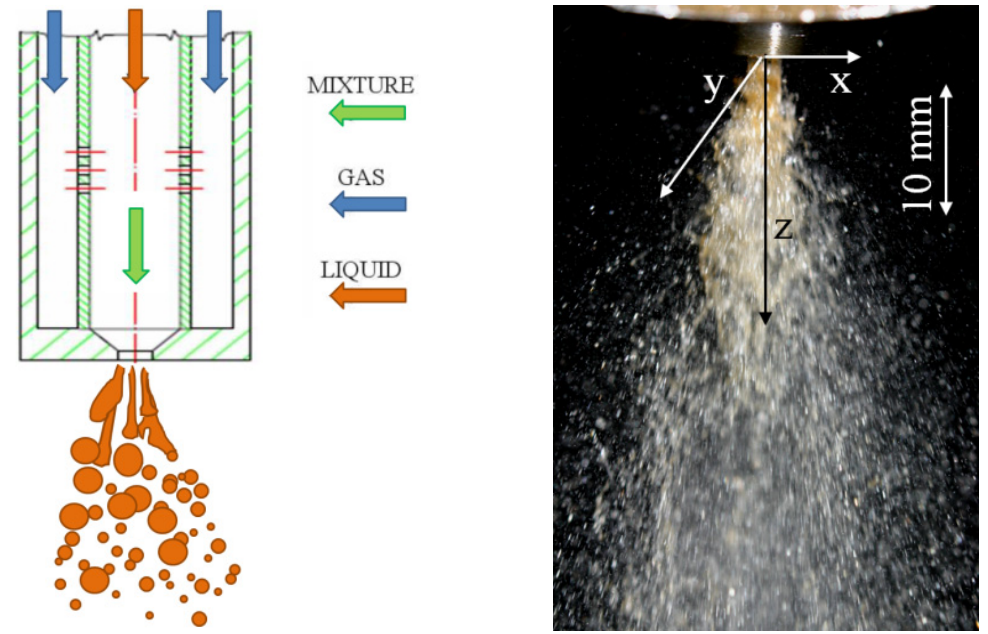

Figure 1: The effervescent atomizer: working principle (left) and real discharge (right).

\section{Results and discussion}

Discharge velocities of the gas and liquid phases in the effervescent spray are estimated first, using a combination of two theoretical two-phase discharge models and measured flow rates. These original velocities are then used in a simple model of droplet dynamics in decaying gas jet for modelling of axial motion of particles with diverse Stokes numbers. Radial profiles of measured mean droplet velocity are compared with theoretical profiles found for a gas jet. And finally a set of size resolved PDA results acquired for a range of operation regimes and positions in the spray are described to document the gas-droplet interaction. 


\subsection{Internal flow and discharge velocity}

Primary break-up and further evolution of the spray strongly depend on the discharge conditions. The liquid with gas form a two-phase mixture which character is driven mainly by GLR. Bubbly flow structure appears at GLR $\leq 6.2 \cdot p_{1}[\%, \mathrm{MPa}][3]$ while annular formation of centrally flowing gas surrounded with liquid exists in the mixing chamber and transports downstream at high GLR. Slug or intermittent structures can form at transition between the two previous flow regimes.

A degree of the mixture homogeneity depends on the flow regime as well as on the upstream conditions - mixing chamber size and aeration process. Liquid always issues with velocity lower than the gas, which is due to character of the discharge of the incompressible liquid and compressible gas. Flowing gas wafts and accelerates the liquid volumes (ligaments, filaments or bulbs) due to a drag force hence decelerating. Rate of its action on the liquid and actual velocity slip depend on the degree of the mixture homogeneity — smaller liquid structures are easier to accelerate.

The discharge conditions of the effervescent atomizer are, for several operation regimes, documented in table 1. Operation pressures, temperatures and flow rates were measured. Discharge velocities were calculated using a combination of two analytical discharge models: Separated Flow Model (SFM) and Homogeneous Flow Model (HFM) without heat transfer (frozen flow) according [9], Appendix 2. Density and liquid/gas surface tension of LHO are $874 \mathrm{~kg} / \mathrm{m}^{3}$ and $0.0297 \mathrm{~kg} / \mathrm{s}^{2}$ respectively. The ratio between these two models was set to satisfy the measured data; typically, depending on the operation conditions, $59-64 \%$ of the total discharged mass corresponded to the SFM and the rest to the HFM.

Table 1: Discharge characteristics.

\begin{tabular}{|l|l|l|l|l|l|}
\hline$p(\mathrm{kPa})$ & 100 & 300 & 300 & 300 & 500 \\
\hline GLR $(-)$ & 0.05 & 0.02 & 0.05 & 0.10 & 0.05 \\
\hline$\dot{m}_{g} *(\mathrm{~g} / \mathrm{s})$ & 0.85 & 0.93 & 1.59 & 2.14 & 2.28 \\
\hline$\dot{m}_{l} *(\mathrm{~g} / \mathrm{s})$ & 16.6 & 47.2 & 32 & 21.4 & 45.6 \\
\hline$w_{g}(\mathrm{~m} / \mathrm{s})$ & 173 & 122 & 170 & 205 & 172 \\
\hline$w_{l}(\mathrm{~m} / \mathrm{s})$ & 21 & 32 & 34 & 36 & 43 \\
\hline$w_{l} / w_{g}(-)$ & 0.12 & 0.27 & 0.20 & 0.18 & 0.25 \\
\hline
\end{tabular}

*measured values, taken from [9].

Resulting velocities are considered as average values within the discharge orifice cross-section. The gas flow and the two-phase flow according HFM is choked at all studied regimes. The liquid reaches only $12-27 \%$ of the gas exit velocity. The liquid-gas velocity ratio, $w_{l} / w_{g}$, is negatively correlated with GLR and positively correlated with inlet pressure; increase in pressure difference accelerates the discharged liquid while choked gas keeps its critical velocity, this effect mainly applies to the fraction of the fluid which follows the SFM. Increase 
in GLR allows for faster discharge of the two-phase mixture fraction which behaves according the HFM due to increased mass of the gas acting on the liquid mass with rising GLR. The gas therefore has to transfer less energy to the liquid and hereby less decelerates.

\subsection{Dynamics of droplets in the spray}

Effervescent sprays can be treated simply as an ensemble of droplets dispersed in a gas flow. To qualitatively describe the velocity field in twin-fluid spray we assume one-way coupling, for the first approximation of the phenomena, and use a simple theoretical case of gas free jet discharged from the nozzle. The gas flow is thought to be an independent jet which acts on the droplets.

\subsubsection{Radial profiles of axial velocity}

Spatially resolved velocity of the in the gas jet according Biswas and Eswaran $[10]$ is

$$
w_{g}(r, z)=\frac{\sqrt{3}}{2} \sqrt{\frac{\sigma J}{\rho_{g} z}}\left(1-\tanh ^{2} \frac{\sigma r}{z}\right)
$$

where $\sigma=7.67$ and $J$ is total momentum of the jet. Awbi [11] suggests:

$$
w_{g}(r, z)=\frac{2.47 w_{g 0}}{\sqrt{z / d_{o}}} \exp \left(-6.7\left(\frac{r}{0.1 z}\right)^{2}\right)
$$

where $w_{g 0}$ is exit centreline velocity and $d_{o}$ is discharge orifice diameter. Both the equations show the velocity decreases according $1 / \sqrt{z}$ and radial velocity profiles are bell-shaped with a maximum in the centreline. Velocity profiles divided by the maximum velocity at given relative radial distance $r_{r}=r / z$ are identical, so the flow is self-similar with $\hat{w}_{g}\left(r_{r}\right)=1-\tanh ^{2} \sigma r_{r}$ according eqn (1) or with $\hat{w}_{g}\left(r_{r}\right)=\exp \left(-6.7\left(10 r_{r}\right)^{2}\right)$ according eqn (2). Note that Panchagnula and Sojka [7] used

$$
\hat{w}_{g}\left(r_{r}\right)=\operatorname{sech}^{2}\left(\sigma r_{r}\right)
$$

as formula for velocity profile of an effervescent spray which is identical to the simplified Biswas's eqn (1).

Description of real velocity field in two-phase jet uses data from PDA droplet measurements. Typical normalised velocity profiles of axial droplet velocity at different axial positions are shown in fig. 2. The values represent an average over all droplet sizes. All the profiles are characterised by similar shape however they tend to narrow with increasing axial distance. The profiles are axisymmetric bellshaped with a maximum in the centreline. Except close the centreline $\left(r_{r}<0.2\right)$ the profiles can be approximated using a power function $\hat{w}_{g}=a r_{r}^{-b}$. The 
coefficient $a$, varies significantly with axial distance which describes the effect of spray narrowing (table 2). The spray behaves as a jet with its origin shifted by about $200 \mathrm{~mm}$ in negative direction. Coefficient $b$ is constant for particular operation regime and slightly varies with change in pressure and GLR. Velocity profiles defined by eqns (1) and (2) differ significantly from the measured spray profiles. These profiles are more flat near the centreline however they decline much faster for $r_{r}>0.2$. Less steep decay of the axial velocity in radial direction for real spray is due to high Stk particles that do not transfer their kinetic energy to the surrounding air as efficiently as the discharged air does. Specific reasons for these differences in gas and droplet velocities in the spray are analysed in the next section in more detail.

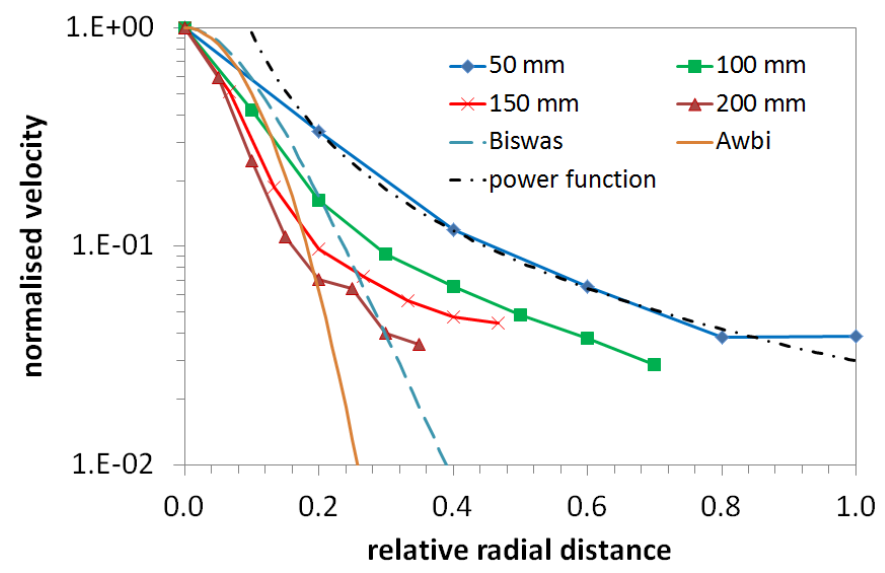

Figure 2: Normalised velocity profiles for atomizer E34 and $p=0.3 \mathrm{MPa}$, $\mathrm{GLR}=5 \%$.

Table 2: Variation of coefficients $\mathrm{a}$ and $\mathrm{b}$ with axial distance and with operation conditions for atomizer E34.

\begin{tabular}{|c|c|c|c|c|c|c|c|c|c|}
\hline$p$ & GLR & \multicolumn{6}{|c|}{$a$} & \multicolumn{4}{|c|}{$b$} \\
\hline$(\mathrm{MPa})$ & $(\%)$ & 50 & 100 & 150 & 200 & 50 & 100 & 150 & 200 \\
\hline 0.1 & 5 & 0.026 & 0.018 & 0.014 & 0.0125 & -1.3 & -1.3 & -1.3 & -1.3 \\
\hline 0.3 & 2 & 0.035 & 0.02 & 0.012 & 0.009 & -1.4 & -1.4 & -1.4 & -1.4 \\
\hline 0.3 & 5 & 0.03 & 0.015 & 0.009 & 0.007 & -1.5 & -1.5 & -1.5 & -1.5 \\
\hline 0.3 & 10 & 0.025 & 0.012 & 0.0075 & 0.006 & -1.5 & -1.5 & -1.5 & -1.5 \\
\hline 0.5 & 5 & 0.031 & 0.014 & 0.0075 & 0.0055 & -1.55 & -1.55 & -1.55 & -1.55 \\
\hline
\end{tabular}

\subsubsection{Variation of velocity in axial direction}

Grant et al. [12] applied exponential function to describe variation of the axial velocity along the spray centreline. Velocity of gas discharged with the velocity $w_{g 0}$ decays with the flight distance $z$ approximately as: 


$$
w_{g}=w_{g 0} \exp \left(-z / Z_{0}\right)
$$

where $Z_{0}$ is the decay constant. The velocity of individual droplets is governed by a momentum equation $[13,14]$, relating the acceleration, $a_{d}$, of a droplet to the relative velocity of the gas to the droplet:

$$
\begin{aligned}
a_{d}=\frac{d w_{d}}{d t} & =-\frac{3}{4} C_{D} \operatorname{Re}_{d} \frac{\mu_{g}}{\rho_{l} C_{c} D_{d}^{2}}\left(w_{d}-w_{g}\right)= \\
& =-\frac{18 \mu_{g}}{\rho_{l} D_{d}^{2}}\left(w_{d}-w_{g}\right)
\end{aligned}
$$

where $\rho_{l}$ and $\mu_{g}$ are particle density and gas dynamic viscosity respectively, the drag coefficient $C_{D}=24 / \operatorname{Re}_{d}$ for $\operatorname{Re}_{\mathrm{d}}<1$ and the Cunningham correction factor $C_{c} \sim 1$ [15], for micron sized particles and larger. This simple approximation assumes one way coupling.

Initial (i.e. discharge) velocities of the gas and liquid phases depend on the operation conditions and internal flow regime (see section 3.1). In the case of very well mixed phases velocity of both the fluids at the moment of discharge can be similar (described by HFM), however in real effervescent sprays the gas flows with higher velocity compared to the liquid. The highest slip between gas and liquid velocities would occur in the case of separated phases (described by SFM). Real slip between the phases will be somewhere between both the limiting cases (SFM, HFM) of the discharge.

A simulation based on eqns (4) and (5) was made for the case where initial (i.e. discharge) velocity of the liquid was set to $20 \%$ of the initial gas velocity, which is a typical case according to table 1. Particle behaviour is expressed using their Stokes number:

$$
S t k=\frac{\rho_{l} D_{d}^{2}\left|\left(w_{d 0}-w_{g 0}\right)\right|}{18 \mu_{g} Z_{0}} .
$$

Using eqns (5) and (6)

$$
a_{d}=-\frac{1}{S t k} \frac{\left|w_{d 0}-w_{g 0}\right|\left(w_{d}-w_{g}\right)}{Z_{0}}
$$

To generalize the results relative velocities $\hat{w}_{g}=w_{g} / w_{g 0}$ and $\hat{w}_{d}=w_{d} / w_{g 0}$ and relative distance $z_{r}=z / Z_{0}$ are used on the corresponding axis in figs. 3 and 4 .

Particles with Stk $<<1$ (represented by particle with $\mathrm{Stk}=0.05$ ) reach the gas velocity in short distance. The relative velocity difference $\left(\left(w_{d}-w_{g}\right) / w_{d 0}\right)$, is originally $80 \%$ and drops down fast due to strong drag force compared to particle inertia. It changes from negative to positive when the $w_{g}$ drops below actual $w_{d}$ and after its positive maximum about $4 \%$ at $z_{r}=0.56$ gradually decreases for larger distances and asymptotically goes to zero. Larger particles need more time to reach the equilibrium with gas flow. Particles with $\mathrm{Stk}=1$ reach maximum 
positive relative velocity slip of $15 \%$ at $z_{r}=1.27$. Position of the velocity slip maximum as well as its amplitude rise with Stk. Large Stk particles (Stk $>10)$ move essentially without change in velocity so their slip velocity is large even in the distances $z_{r}>2$ (which correspond to $z>300 \mathrm{~mm}$ in real spray and are thus out of the typical area of interest).

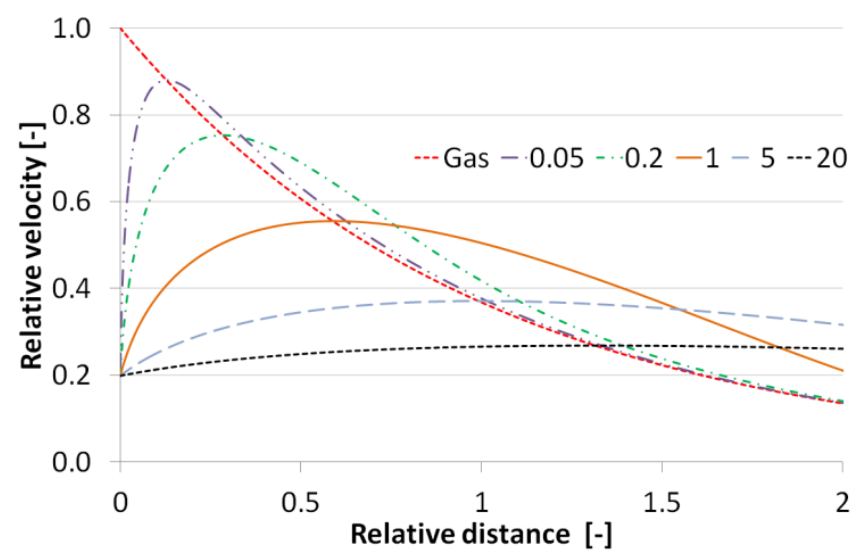

Figure 3: Normalized velocity of gas and particles with different original Stk.

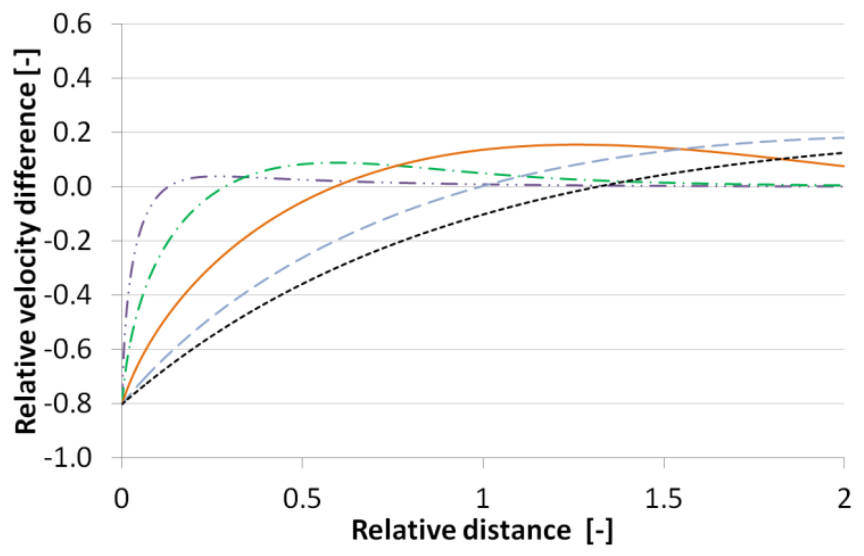

Figure 4: Normalized difference between gas and particle velocity.

\subsection{Size resolved PDA data}

Experimental data acquired in spray of the effervescent atomizer E34 using PDA are summarised in table 3. Mean velocities, fluctuating velocities and Stokes numbers are documented for several size classes for several operation regimes 
Table 3: Size resolved characteristics of droplets for different operation conditions and positions in the spray.

\begin{tabular}{|c|c|c|c|c|c|c|c|c|c|c|}
\hline$p$ & GLR & $r$ & $z$ & value & \multicolumn{6}{|c|}{ Droplet diameter [microns] } \\
\hline (MPa) & $(\%)$ & $(\mathrm{mm})$ & $(\mathrm{mm})$ & $(\mathrm{m} / \mathrm{s},-)$ & 5 & 10 & 20 & 30 & 50 & 100 \\
\hline 0.1 & 2 & 0 & 50 & $v_{\text {mean }}$ & 15.4 & 14.9 & 15.0 & 15.6 & 16.4 & 16.7 \\
\hline 0.1 & 2 & 0 & 50 & $v_{r m s}$ & 2.49 & 2.84 & 2.79 & 2.79 & 2.33 & 2.24 \\
\hline 0.1 & 2 & 0 & 50 & Stk & 0.014 & 0.086 & 0.315 & 0.415 & 0.168 & 1.277 \\
\hline 0.3 & 5 & 0 & 50 & $v_{\text {mean }}$ & 62.8 & 63.1 & 65.7 & 69.6 & 69.9 & 59.2 \\
\hline 0.3 & 5 & 0 & 50 & $v_{r m s}$ & 14.85 & 14.95 & 15.05 & 15.13 & 14.52 & 11.58 \\
\hline 0.3 & 5 & 0 & 50 & Stk & 0.04 & 0.142 & 0.014 & 1.807 & 5.527 & 35.02 \\
\hline 0.5 & 10 & 0 & 50 & $v_{\text {mean }}$ & & & 95.6 & 101.4 & 95.8 & \\
\hline 0.5 & 10 & 0 & 50 & $v_{r m s}$ & & & 12.66 & 15.36 & 14.07 & \\
\hline 0.5 & 10 & 0 & 50 & Stk & & & 0 & 2.755 & 0.248 & \\
\hline 0.5 & 10 & 10 & 50 & $v_{\text {mean }}$ & 24.8 & 26.5 & 25.9 & 26.6 & 32.7 & 39.0 \\
\hline 0.5 & 10 & 20 & 100 & $v_{\text {mean }}$ & 9.3 & 10.0 & 8.7 & 8.1 & 8.5 & 20.8 \\
\hline 0.5 & 10 & 30 & 150 & $v_{\text {mean }}$ & 3.6 & 3.6 & 2.7 & 3.1 & 3.6 & 4.4 \\
\hline 0.5 & 10 & 40 & 200 & $v_{\text {mean }}$ & 1.9 & 1.8 & 1.1 & 1.7 & 2.1 & 2.6 \\
\hline 0.5 & 10 & 10 & 50 & $v_{r m s}$ & 9.51 & 11.07 & 11.62 & 11.75 & 11.28 & 8.32 \\
\hline 0.5 & 10 & 20 & 100 & $v_{r m s}$ & 5.70 & 6.70 & 5.82 & 5.23 & 5.33 & 6.96 \\
\hline 0.5 & 10 & 30 & 150 & $v_{r m s}$ & 3.11 & 2.76 & 2.63 & 2.67 & 2.93 & 3.46 \\
\hline 0.5 & 10 & 40 & 200 & $v_{r m s}$ & 1.55 & 2.12 & 1.06 & 1.97 & 1.75 & 2.26 \\
\hline 0.5 & 10 & 10 & 50 & Stk & 0 & 0.088 & 0.212 & 0.813 & 10.29 & 73.98 \\
\hline 0.5 & 10 & 20 & 100 & Stk & $1 \mathrm{E}-4$ & 0.02 & 0.061 & 0.262 & 0.463 & 30.17 \\
\hline 0.5 & 10 & 30 & 150 & Stk & 0.002 & 0.007 & 0.089 & 0.14 & 0.145 & 0.799 \\
\hline 0.5 & 10 & 40 & 200 & Stk & 3E-4 & 0.001 & 0.042 & 0.024 & 0.06 & 0.931 \\
\hline 0.5 & 10 & 20 & 50 & $v_{\text {mean }}$ & 8.3 & 8.5 & 7.1 & 7.2 & 8.2 & 17.2 \\
\hline 0.5 & 10 & 30 & 50 & $v_{\text {mean }}$ & 8.7 & & 7.9 & 3.3 & 3.1 & 12.1 \\
\hline 0.5 & 10 & 40 & 50 & $v_{\text {mean }}$ & & & 5.2 & 3.3 & 1.5 & 11.5 \\
\hline 0.5 & 10 & 60 & 50 & $v_{\text {mean }}$ & & & & & 1.3 & 7.3 \\
\hline 0.5 & 10 & 20 & 50 & $v_{r m s}$ & 5.71 & 7.60 & 4.74 & 5.16 & 5.67 & 4.29 \\
\hline 0.5 & 10 & 30 & 50 & $v_{r m s}$ & 6.69 & & 4.79 & 2.90 & 3.08 & 2.44 \\
\hline 0.5 & 10 & 40 & 50 & $v_{r m s}$ & & & 4.72 & 3.19 & 1.50 & 1.08 \\
\hline 0.5 & 10 & 60 & 50 & $v_{r m s}$ & & & & & 0.92 & 0.50 \\
\hline 0.5 & 10 & 20 & 50 & Stk & 0 & 0.011 & 0.235 & 0.455 & 0.043 & 44.01 \\
\hline 0.5 & 10 & 30 & 50 & Stk & 0 & & 0.149 & 2.233 & 6.409 & 15.29 \\
\hline 0.5 & 10 & 40 & 50 & Stk & & & 0 & 0.736 & 3.914 & 25.95 \\
\hline 0.5 & 10 & 60 & 50 & Stk & & & & & 0 & 20.51 \\
\hline
\end{tabular}

(inlet pressures and GLR values) and positions in the spray (radial and axial distances).

The results in each particular conditions and place are made typically for 50,000 particles as given in fig. 5 which shows illustrative size-velocity correlations. Individual particles are represented by red dots, 50-particle flowing average and \pm standard deviation are displayed as well. 

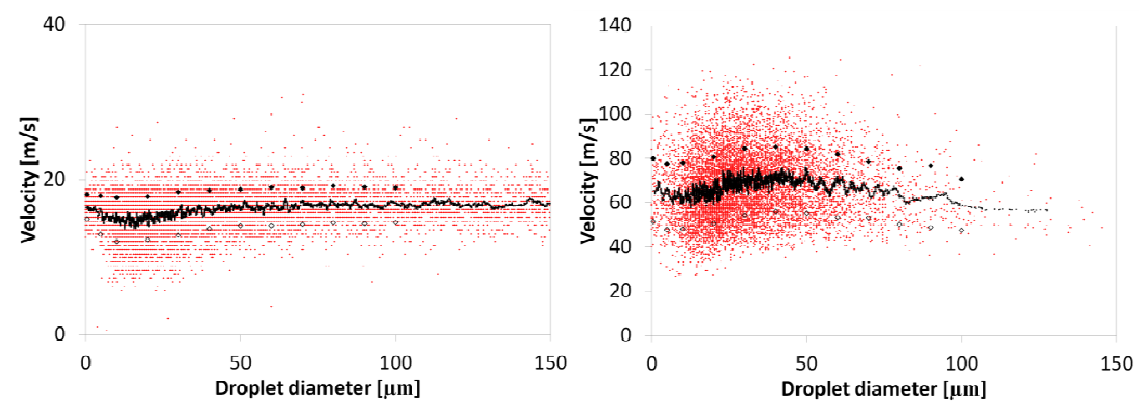

Figure 5: Diameter-velocity correlation, $r_{r}=0, z=50 \mathrm{~mm}$, left: $p=0.1 \mathrm{MPa}$, GLR $=2 \%$, right: $p=0.3 \mathrm{MPa}, \mathrm{GLR}=5 \%$.

Mean velocity varies with particle size within a range of several m/s typically. This variation is closely related with particle Stokes number. Variation of mean velocity with operation pressure and GLR can be explained with discharge conditions (see table 1). Higher pressures and higher GLR lead to higher discharge velocities that are reflected in the spray downstream. Radial profiles of velocity are approximately bell-shaped as already described in fig. 2 (without the size discrimination). The decay of mean velocity with axial distance is obvious. However, it does not correspond quantitatively to the theoretical decay (fig. 3), which is steeper.

Fluctuating (rms) velocity of particles reflects two phenomena: response of the particles during their flow and evolution to turbulent fluctuations of the gas and also velocity fluctuations imposed to the liquid during discharge. The rms velocity does not vary strongly with particle size but depend well on operation conditions and on position in the spray. Its deep analysis and discrimination between their two sources would require larger data set and it is out of range of this paper.

Stokes numbers of the droplets are generally much lower than unity for particles up to $10 \mu \mathrm{m}$ and these particles thus follow the gas flow smoothly. Stk for particles with $10 \mu \mathrm{m}<D_{d}<50 \mu \mathrm{m}$ depends on the flow regime and position in the spray and can be found typically within a range $0.1<\mathrm{Stk}<10$. Particles with $D_{d}=100 \mu \mathrm{m}$ and larger have Stk usually larger than 10 and can be classified as high inertia particles, which very weakly interact with the gas. These particles conserve most of the original inertia of the liquid when discharged.

\section{Conclusions}

Discharge of the liquid-gas flow from an effervescent nozzle was found choked for all studied operation regimes. It was described using a combination of two discharge models, SFM and HFM and typically, depending on the operation conditions, 59-64\% of the total discharged mass corresponded to the SFM and the rest to the HFM. Discharge velocity of the liquid was found to be only 12 
$27 \%$ of the gas exit velocity depending on operation conditions. The liquid-gas velocity ratio is negatively correlated with GLR and positively correlated with inlet pressure.

A simple model was used to predict droplet motion in a gas jet with exponential velocity decay. It shows particles with $\mathrm{Stk}<<1$ reach the gas velocity in very short distance, get slightly faster and then converges the decelerated gas. Position of the maximum of gas-liquid velocity slip as well as its amplitude increase with

Stk. Particles with Stk $>10$ move essentially without velocity change. The decay of mean axial velocity with axial distance in real spray is obvious; however it does not quantitatively correspond to theoretical decay, which is steeper. Radial profiles of axial droplet velocity, as measured using PDA, are axisymmetric bell-shaped with a maximum in the centreline analogous to the profile defined for a simple gas jet which however are more flat near the centreline and decline much faster for higher radial positions.

Mean velocity in particular spray position varies with particle size within a range of several $\mathrm{m} / \mathrm{s}$ typically. This variation is closely related with particle Stokes number. Variation of mean velocity with operation pressure and GLR can be explained with discharge conditions; higher pressures and GLRs lead to higher discharge velocities that are reflected in the spray downstream.

Stokes numbers are generally much lower than unity for particle sizes $D_{d}$ up to $10 \mu \mathrm{m}$ so these particles smoothly follow the gas flow. Stk for size $10 \mu \mathrm{m}<D_{d}<50 \mu \mathrm{m}$ depends on flow regime and position in the spray and can be found typically within a range $0.1<$ Stk $<10$. Particles with $D_{d} \geq 100 \mu \mathrm{m}$ have usually Stk $>10$ and very weakly interact with the gas.

\section{Acknowledgement}

The authors greatly acknowledge financial support from project No. 101/11/1264 funded by the Czech Grant Agency.

\section{References}

[1] Lefebvre, A. H., Wang, X. F. and Martin, C. A. (1988) Spray Characteristics of Aerated-Liquid Pressure Atomizers. Journal of Propulsion and Power. 4(4) 293-298.

[2] Sovani, S. D., Sojka, P. E. and Lefebvre, A. H. (2001) Effervescent atomization. Progress in Energy and Combustion Science. 27(4) 483-521.

[3] Jedelsky, J. and Jicha, M. Energy conversion during effervescent atomization. Fuel (0).

[4] Sovani, S. D., Crofts, J. D., Sojka, P. E., Gore, J. P. and Eckerle, W. A. (2005) Structure and steady-state spray performance of an effervescent diesel injector. Fuel. 84(12-13) 1503-1514.

[5] Lund, M. T., Sojka, P. E., Lefebvre, A. H. and Gosselin, P. G. (1993) Effervescent atomization at low mass flow rates. Part I: the influence of surface tension. 3(1) 77-89. 
[6] Lefebvre, A. H. (1996) Some recent developments in twin-fluid atomization. Particle \& Particle Systems Characterization. 13(3) 205-216.

[7] Panchagnula, M. V. and Sojka, P. E. (1999) Spatial droplet velocity and size profiles in effervescent atomizer-produced sprays. Fuel. 78(6) 729741.

[8] Santangelo, P. J. and Sojka, P. E. (1995) A holographic investigation of the near-nozzle structure of an effervescent atomizer-produced spray. Atomization and Sprays. 5(2) 137-155.

[9] Jedelsky, J., Jicha, M., Slama, J. and Otahal, J. (2009) Development of an Effervescent Atomizer for Industrial Burners. Energy \& Fuels. 23 61216130.

[10] Biswas, G. and Eswaran, V. (2002) Turbulent flows: fundamentals, experiments and modeling CRC Press; Narosa Pub. House, Boca Raton, Fla. New Delhi.

[11] Awbi, H. B. (2003) Ventilation of buildings 2nd ed Spon Press, London; New York.

[12] Grant, P. S., Cantor, B. and Katgerman, L. (1993) Modeling of droplet dynamic and thermal histories during spray forming 1 . Individual droplet behavior. Acta Metallurgica et Materialia. 41(11) 3097-3108.

[13] Srivastava, A. K., Ojha, S. N. and Ranganathan, S. (1998) Microstructural features and heat flow analysis of atomized and spray-formed Al-Fe-V-Si alloy. Metallurgical and Materials Transactions a - Physical Metallurgy and Materials Science. 29(8) 2205-2219.

[14] Szekely, J. (1979) Fluid flow phenomena in metals processing Academic Press, New York.

[15] Hinds, W. C. (1999) Aerosol technology: properties, behavior, and measurement of airborne particles 2nd ed Wiley, New York. 